\title{
ARMA Signals with Specified Symmetric Marginal Probability Distribution
}

\author{
Bernard Picinbono, Life Fellow, IEEE
}

\begin{abstract}
Except in the case of normal (i.e Gaussian) distribution, it is very difficult to calculate the marginal probability distribution of ARMA signals. By using a particular form of modeling such signals with random coefficients we show that the problem can be solved and we present an algorithm allowing the numerical generation of ARMA signals with arbitrary specified marginal distribution function. Their properties are analyzed in computer experiments. The experimental results show in general a very good agreement with theoretical calculations.
\end{abstract}

\section{Index Terms}

ARMA signals, autoregressive signals, moving average signals, linear prediction, innovation, probability distribution, random coefficients, Bernoulli signals, non Gaussian signals and noise, signal and noise modeling, statistical signal analysis.

\section{INTRODUCTION}

ARMA signals are probably the most important models of random processes used in various fields of Signal Processing such as, for example, spectral analysis, estimation and prediction [1], [2]. This comes partially from the simplicity of their definition. Indeed a real strictly ARMA signal is the output of a real dynamical filter when its input is a real strictly white noise. A discrete time dynamical filter is a filter which is stable, causal and such that its transfer function (TF) is a rational function, $i$. $e$. a ratio

\footnotetext{
Manuscript received June 15, 2009.
}

Copyright (c) 2008 IEEE. Personal use of this material is permitted. However, permission to use this material for any other purposes must be obtained from the IEEE by sending a request to pubs-permissions@ieee.org. B. Picinbono is with the Laboratoire des Signaux et Systèmes (L2S), a joint laboratory of the C.N.R.S. and the École Supérieure d'Électricité, Plateau de Moulon, 3 rue Joliot-Curie 91192, Gif sur Yvette, France. The L2S is associated with the University of Paris-Sud, France. E-mail: bernard.picinbono@1ss.supelec.fr, . 
of two polynomials. The causality and stability assumptions imply that the poles of this rational fraction are located inside the unit circle. Furthermore a discrete time strictly white noise is a sequence of IID random variables (RV) $W_{k}$ and the statistical properties of such a noise are entirely defined from the distribution function (DF) $F_{W}(w)$ common to all these RVs. As a result an ARMA signal is defined by the zeros and poles of its corresponding TF, which is equivalent to the coefficients of the polynomials of the numerator and the denominator, and by the DF $F_{W}(w)$.

The second-order properties of ARMA signals are perfectly known, even if the calculations are sometimes rather complicated. The $z$-transform of the covariance function of an ARMA signal is then $\Gamma(z)=H(z) H\left(z^{-1}\right) \sigma_{W}^{2}$, where $H(z)$ is the TF of the filter and $\sigma_{W}^{2}$ the variance of the driving noise. The covariance function can then be directly deduced either from the poles and zeros of the TF or from the coefficients of the two polynomials defining this TF.

There are however problems of statistical signal processing where the DF of the signal must be known. For these problems ARMA modeling is not well adapted, except in the very important case where the input signal is a white Gaussian noise (WGN). Indeed in this case the ARMA signal is also Gaussian, and all its statistical properties are defined from those of the second order introduced above. If, on the contrary, the $\mathrm{DF} F_{W}(w)$ is not a normal distribution it is difficult, and often impossible, to calculate explicitly the DF of the resulting ARMA signal.

The purpose of this paper is to overcome this difficulty and to introduce a class of signals having the same second order properties as ARMA signals, but such that their marginal DF is specified in advance. For this we introduce polynomials with random coefficients with specific properties. In order to simplify the presentation we shall study separately AR and MA signals.

Note that similar problems have been already discussed in a completely different context [3], [4], [5]. The methods proposed in these papers start from a Gaussian white signal transformed by a linear filter followed by an instantaneous nonlinear transformation. The calculations however are rather complicated and the condition of positive definiteness of the covariance function is not always satisfied. These papers study a general problem, without using the specifications of AR or MA signals, which is the main purpose of what follows.

\section{ARRC MODELS}

\section{A. Review of known results on AR signals}

A strictly autoregressive signal of order $r[\operatorname{AR}(r)] \bar{X}_{k}$ is a discrete time signal defined by the recursion

$$
\bar{X}_{k}=\mathbf{a}^{T} \mathbf{X}_{k}+W_{k},
$$


where $\mathbf{a}$ is the regression vector with components $a_{1}, a_{2}, \ldots, a_{r}$ and $\mathbf{X}_{k}$ the finite past vector of $\bar{X}_{k}$ with components $\bar{X}_{k-1}, \bar{X}_{k-2}, \ldots, \bar{X}_{k-r}$, while $W_{k}$ is a strictly white noise. When, however, one is interested only in second order properties of $\bar{X}_{k}$, the assumption of independence of the $W_{k}$ s can be omitted and a weakly white noise, i.e. a sequence of uncorrelated RVs is sufficient. In this case the resulting signal is called weakly autoregressive of order $r$.

The recursion (1) means that $W_{k}$ is filtered in a dynamical filter with the $\mathrm{TF} H(z)=z^{r} / D(z)$ where $D(z)=z^{r}-\left(a_{1} z^{r-1}+a_{2} z^{r-2}+\ldots+a_{r}\right)$. In order to obtain a signal $\bar{X}_{k}$ with finite variance (stability problem), the vector a must belong to a domain called stability domain ensuring that the roots of $D(z)$ are located inside the unit circle. This domain is presented in Fig. 1 when $r=2$. It is the interior of the triangle $\mathrm{ABC}$ of the plane $a_{1} \times a_{2}$. The parabola $\mathrm{COB}$ defined by the equation $a_{2}=-a_{1}^{2} / 4$ makes a separation between the parts of the stability domain corresponding to real and complex roots of $D(z)$. If $a_{2}<-a_{1}^{2} / 4$, the two roots have the form $z_{ \pm}=m \exp ( \pm j \phi)$, with $0<m<1$.

One of the most important properties of AR signals appears in prediction theory. Let $\hat{\bar{X}}_{k}$ be the onestep mean square prediction with infinite past of $\bar{X}_{k}$, which is the mean square estimation of $\bar{X}_{k}$ in terms of all the past values $\bar{X}_{k-l}, l>0$. The fact that $W_{k}$ in (1) is strictly white implies that $\hat{\bar{X}}_{k}=\mathbf{a}^{T} \mathbf{X}_{k}$, which means that whatever the probability distribution of the driving noise $W_{k}$ may be, the mean square prediction is linear.

On the other hand the linearity of the mean square prediction does not imply that $W_{k}$ is strictly white. This prediction indeed is the conditional expectation value $\mathrm{E}\left[\bar{X}_{k} \mid P_{k}\left(\left\{\bar{X}_{i}\right\}\right)\right]$, where $P_{k}\left(\left\{\bar{X}_{i}\right\}\right)$ is the collection of past RVs $\bar{X}_{k-i}, i>0$. It results immediately from (1) that $P_{k}\left(\left\{\bar{X}_{i}\right\}\right)=P_{k}\left(\left\{W_{i}\right\}\right)$ because the past values of $W_{k}$ can be deduced from those of $\bar{X}_{k}$. As a consequence if the mean square prediction $\mathrm{E}\left[\bar{X}_{k} \mid P_{k}\left(\left\{\bar{X}_{i}\right\}\right)\right]=\mathbf{a}^{T} \mathbf{X}_{k}$, then $\mathrm{E}\left[W_{k} \mid P_{k}\left(\left\{W_{i}\right\}\right)\right]=0$. This means that $W_{k}$ is a strongly white noise, as defined on p. 254 of [1]. As a consequence we say that $\bar{X}_{k}$ is a strongly AR(r) signal.

Finally, if $W_{k}$ is only weakly white, which means that the RVs $W_{k}$ are only uncorrelated, then the property of linearity of the conditional expectation value has no reason to be true, yet $\mathbf{a}^{T} \mathbf{X}_{k}$ remains the best linear mean square prediction of $X_{k}$. In this case we say that $X_{k}$ is a weakly $A R(r)$ signal.

\section{B. ARRC(2) Signals}

We shall now introduce the concept of autoregressive with random coefficients [ARRC(2)] signals. This idea was presented in a old book which contains numerous references of papers on this subject [6]. Much more recent references can be found in [7]. The approaches of all these papers, however, are quite different compared to the one presented below. 
Let $a_{1}$ and $a_{2}$ be two real numbers satisfying the condition $\left|a_{1}\right|+\left|a_{2}\right|<1$, which means that the point $\left(a_{1}, a_{2}\right)$ is located inside the square ADEF of Fig. 1. Let us also introduce

$$
\epsilon_{1}=a_{1} /\left|a_{1}\right|=\operatorname{sign}\left(a_{1}\right) ; \epsilon_{2}=a_{2} /\left|a_{2}\right|=\operatorname{sign}\left(a_{2}\right) .
$$

Let $\operatorname{rect}(a, b ; x)$ be the "rectangular" (or window) function defined by

$$
\operatorname{rect}(a, b ; x)=1 \text { if } a<x \leq b,
$$

and 0 otherwise. Consider now the three functions $\psi_{i}(x)$ defined by

$$
\psi_{1}(x)=\operatorname{rect}\left(0,\left|a_{1}\right| ; x\right), \psi_{2}(x)=\operatorname{rect}\left(\left|a_{1}\right|,\left|a_{1}\right|+\left|a_{2}\right| ; x\right), \psi_{3}(x)=\operatorname{rect}\left(\left|a_{1}\right|+\left|a_{2}\right|, 1 ; x\right) .
$$

These three functions yield a partition of the interval $[0,1]$ in three domains of measure $\left|a_{1}\right|,\left|a_{2}\right|$ and $a_{3}$ with $a_{3}=1-\left|a_{1}\right|-\left|a_{2}\right|$. The condition on $\left|a_{i}\right|$ introduced above ensures that $a_{3}>0$. As a consequence of (4), these functions satisfy $\psi_{i}(x) \psi_{j}(x)=0$ when $i \neq j$.

Let now $B_{k}$ be a uniform strictly white noise in which the RVs $B_{k}$ are IID and uniformly distributed in the interval $[0,1]$, and consider the signal $X_{k}$ defined by the recursion

$$
X_{k}=\epsilon_{1} \psi_{1}\left(B_{k}\right) X_{k-1}+\epsilon_{2} \psi_{2}\left(B_{k}\right) X_{k-2}+\psi_{3}\left(B_{k}\right) W_{k},
$$

where $W_{k}$ is a symmetric strictly white noise. This means that the characteristic function $\phi_{W}(u)$ of $W_{k}$ is an even function of $u$ and then, when there exists a probability density function $(\operatorname{PDF}) p_{W}(w)$, this $\mathrm{PDF}$ is an even function of $w$. Suppose also that the RVs $W_{k}$ and $B_{l}$ are independent. Equation (5) means that $X_{k}$ is equal to $\epsilon_{1} X_{k-1}$ with the probability $\left|a_{1}\right|$, to $\epsilon_{2} X_{k-2}$ with the probability $\left|a_{2}\right|$ and to $W_{k}$ with the probability $a_{3}$.

When considering a model like (5), the first question that occurs is its stability. This question exists for all the random signals defined by a recursion from a white noise. This is for example the case when using bilinear models introduced in the standard book of Granger [8]. But this is also one important topic in the analysis of autoregressive models with random coefficients [6], [7], [9], [10]. The question is in general rather complicated, but the structure of the random coefficients in the particular model (5) introduces important simplifications.

The stability problem consists in verifying whether or not the recursion (5) defines a second-order signal, which means a signal with finite first and second order moments for any $k$.

Let us first consider the case of the mean value. Because of the symmetry assumption introduced above, the driving noise $W_{k}$ is zeromean valued. Furthermore it results from the structures of the functions $\psi_{i}($. that $\mathrm{E}\left[\psi_{i}\left(B_{k}\right)\right]=\left|a_{i}\right|$ for $i=1$ or 2 . As a consequence the moments $m_{k}=\mathrm{E}\left(X_{k}\right)$ satisfy $m_{k}=$ 
$a_{1} m_{k-1}+a_{2} m_{k-2}$. This equation means that $m_{k}$ is the output of an all poles dynamical filter with a zero input. This implies that, whatever the initial conditions $m_{1}$ and $m_{2}$ are, if the coefficients $a_{1}$ and $a_{2}$ are the coordinates of a point located in the stability domain of Fig. 1, the moments $m_{k}$ tend to 0 when $k \rightarrow \infty$.

Let us now consider the case of the second-order moments. For simplification suppose that the the initial moments in the recursion of the moments are zero, which implies that $m_{k}=0$ for all $k$. In order to calculate the variance $\sigma_{k}^{2}$ of $X_{k}$ defined by (5) we use the facts that the functions $\psi_{i}($.$) defined by (4)$ satisfy $\psi_{i}(x)=\psi_{i}^{2}(x)$ and that their product is zero, as indicated above. As a result we obtain

$$
\sigma_{k}^{2}=\left|a_{1}\right| \sigma_{k-1}^{2}+\left|a_{2}\right| \sigma_{k-2}^{2}+a_{3} \sigma_{W}^{2}
$$

where $\sigma_{W}^{2}$ is the variance of $W_{k}$. If the $a_{i}$ s are the coordinates of a point located inside the square ADEF of Fig. 1, the same property is valid for the point with coordinates $\left|a_{i}\right|$. This implies that $\sigma_{k}^{2}$ is the output of a stable all poles filter with the constant input $a_{3} \sigma_{W}^{2}$. Then it results from the definition of $a_{3}$ that, whatever the initial conditions are, $\sigma_{k}^{2}$ tends to $\sigma_{W}^{2}$ when $k \rightarrow \infty$. This shows that the signal (5) is stable for its first and second-order moments.

The ARRC(2) signals have two fundamental properties which are the origin of their interest in the discussion that follows.

Property 1. Let $X_{k}$ be an ARRC(2) stable signal defined by the coefficients $a_{1}$ and $a_{2}$ and by the strictly white noise $W_{k}$. Let $\bar{X}_{k}$ be the classical strictly AR(2) signal defined by the same parameters. If the marginal probability distribution of $W_{k}$ is symmetric, then $X_{k}$ and $W_{k}$ have the same marginal probability distribution.

Proof. Let $\phi_{W}(u)$ and $\phi_{k}(u)$ be the characteristic functions (CF) of $W_{k}$ and $X_{k}$ respectively. The CF of $X_{k}$ is $\mathrm{E}\left[\exp \left(j u X_{k}\right)\right]$. Using (5) and taking the expectation with respect to the variable $B_{k}$ yields

$$
\phi_{k}(u)=\left|a_{1}\right| \mathrm{E}\left[\exp \left(j u \epsilon_{1} X_{k-1}\right)\right]+\left|a_{2}\right| \mathrm{E}\left[\exp \left(j u \epsilon_{2} X_{k-2}\right)\right]+a_{3} \mathrm{E}\left[\exp \left(j u W_{k}\right)\right] .
$$

This can be transformed into a recursion of the CFs that is

$$
\phi_{k}(u)=\left|a_{1}\right| \phi_{k-1}\left(\epsilon_{1} u\right)+\left|a_{2}\right| \phi_{k-2}\left(\epsilon_{2} u\right)+a_{3} \phi_{W}(u) .
$$

It is clear that if $\phi_{i-1}(u)=\phi_{i-2}(u)=\phi_{W}(u)$, then $\phi_{k}(u)=\phi_{W}(u), \forall k \geq i$, because $\left|a_{1}\right|+\left|a_{2}\right|+a_{3}=1$. Suppose now that the initial conditions of this recursion are $\phi_{1}(u)$ and $\phi_{2}(u)$. As the roots of the polynomial $z^{2}-\left|a_{1}\right| z-\left|a_{2}\right|$ are inside the unit circle, because of the stability assumption, then the solution of the recursion is a sum of two terms. The first, depending on the initial conditions, corresponds 
to the transient regime and tends to 0 when $k \rightarrow \infty$, and the second corresponds to the permanent regime which is $\phi_{k}(u)=\phi_{W}(u)$, because of the definition of $a_{3}$. But it is clear in Fig. 1 that if the point $a_{1}, a_{2}$ is inside the square ADEF, this is also valid for the point $\left|a_{1}\right|,\left|a_{2}\right|$. As a consequence for any arbitrary initial conditions $\phi_{1}(u), \phi_{2}(u)$ we have $\phi_{k}(u) \rightarrow \phi_{W}(u)$ when $k \rightarrow \infty$.

In practice this convergence is very fast and when using sequence of say $10^{6}$ values of $X_{k}$, the influence of the initial conditions is negligible. Note finally that (6) is a direct consequence of (8).

This result is interesting because, as indicated above, the marginal PDF of a classical AR(2) signal is very difficult to obtain, except in the Gaussian case, while for an ARRC(2) signal satisfying the assumptions introduced it is obtained without any calculation and is equal to the one of the noise $W_{k}$.

Property 2. Under the same assumptions as for Property 1, the signals $X_{k}$ and $\bar{X}_{k}$ defined by (5) and (1) respectively, with $\mathbf{a}=\left[a_{1}, a_{2}\right]^{T}$ and $r=2$, have the same normalized covariance functions.

Proof. As indicated above it results from the symmetry assumption that the signal $X_{k}$ is zeromean valued. Let us now consider its second order properties. They are described by the covariance function $\gamma_{p}=\gamma[p]=\mathrm{E}\left(X_{k} X_{k-p}\right)$. The use of (5) leads to the calculation of $\mathrm{E}\left\{\left[\epsilon_{1} \psi_{1}\left(B_{k}\right) X_{k-1}\right] X_{k-p}\right\}$. Taking into account the previous definitions, this expectation is equal to $\epsilon_{1}\left|a_{1}\right| \gamma_{p-1}=a_{1} \gamma_{p-1}$. After the same calculation on the second term of the RHS of (5) we obtain

$$
\gamma_{p}=a_{1} \gamma_{p-1}+a_{2} \gamma_{p-2}
$$

which is the same recursion as the one obtained for a classical $\operatorname{AR}(2)$ signal defined by the two coefficients $a_{1}$ and $a_{2}$. It is clear that the variances of $X_{k}$ and $\bar{X}_{k}$ have no reason to be the same, and it is easy to verify that they are effectively different. But it results from the recursion (9) that their normalized covariance functions, ratios of the covariance function and the variance, are the same.

\section{Extensions to ARR(r) Signals}

The extension of this procedure to orders higher than 2 is in its principle straightforward. The only problem is to insert in the stability domain the condition $\left|a_{1}\right|+\left|a_{2}\right|+\ldots+\left|a_{r}\right|<1$. As the stability domain of an all poles dynamical filter is easier to define by using the so-called reflection coefficients $k_{i}$ appearing in the lattice structure of the filter [1], [2], than by using the regression coefficients $a_{i}$ appearing in (1), we shall use these reflection coefficients in the following analysis.

By using these regression coefficients we introduce the same procedure as for the case of AR(2) signals. 
The signal $X_{k}$ is then now defined by

$$
X_{k}=\sum_{i=1}^{r} \epsilon_{i} \psi_{i}\left(B_{k}\right) X_{k-i}+\psi_{r+1}\left(B_{k}\right) W_{k},
$$

where the functions $\psi_{i}(x)$ are defined by an obvious extension of those of (4).

It is easy to verify that the properties 1 and 2 introduced in the previous subsection concerning ARRC(2) models are still valid and the proofs are exactly the same.

\section{Computer Simulations and Experiments}

We shall now present results of computer simulations and experiments illustrating the previous theoretical results.

1) Order 2: In all the results reported below the parameters $a_{1}, a_{2}$ are the same. According to (9) and to Property 2 this means that all our signals have the same normalized covariance function. The values of the regression parameters are $a_{1}=0.2$ and $a_{2}=-0.5$. This implies that the point $\left(a_{1}, a_{2}\right)$ is located inside the domain allowing for the definition of an ARRC(2) signal and that the roots of the polynomial $D(z)$ introduced above are $m \exp ( \pm j 2 \pi s)$ with $m=0.7071$ and $s=0.2274$.

The differences between the various signals studied below come then uniquely from the PDFs $p_{W}(w)$ of the driving noise $W_{k}$. In order to verify Property 1 one example of PDF could be sufficient. We prefer however to use several examples of possible PDFs $p_{W}(w)$ with very different structures to verify that these properties are effectively independent of the nature of each PDF and not due to a specific structure that could appear in one single example.

We shall begin our analysis by covariance measurements. Our aim is to show that the normalized covariance function of the ARRC(2) signal studied is the same as the one of the AR(2) signal defined by the same coefficients $a_{1}$ and $a_{2}$. Instead of measuring directly the covariance function which is a decreasing function but with a great number of non-vanishing values, we shall use the point that two signals with the same normalized covariance function introduce the same structure of their innovation in the one-step linear prediction with infinite past. The reason is that this structure is entirely defined from the normalized covariance function. The innovation of the classical AR(2) signal $\bar{X}_{k}$ defined by (1) and $a_{1}$ and $a_{2}$ is $\tilde{\bar{X}}_{i}=\bar{X}_{i}-a_{1} \bar{X}_{i-1}-a_{2} \bar{X}_{i-2}$. It is clear that $\tilde{\bar{X}}_{k}=W_{k}$, which is the white driving noise of the model. It is then sufficient to show that the signal

$$
\tilde{X}_{k} \triangleq X_{k}-a_{1} X_{k-1}-a_{2} X_{k-2}
$$

where $X_{k}$ is given by (5) is a weakly white noise, whatever the probability distribution of $W_{k}$. 
The first step of the simulation is then to generate a sequence of $N$ values of IID RVs $W_{k}$ with a specified symmetric distribution. For this we start from a sequences of $N$ RVs $A_{k}$ IID with a uniform distribution in $[0,1]$, which can be obtained by various programs of simulation. By using appropriate transformations of $A_{k}$ we obtain another sequence $W_{k}$ of RVs that are still IID and with the PDF $p_{W}(w)$. The symmetric PDFs used in what follows are

$$
\begin{aligned}
& p_{1}(w)=(1 / 2) \exp (-|w|), \\
& p_{2}(w)=\operatorname{rect}(-1 / 2,+1 / 2 ; w), \\
& p_{3}(w)=\operatorname{rect}(-1,+1 ; w)(1 / \pi)\left(1-w^{2}\right)^{-1 / 2}, \\
& p_{4}(w)=\operatorname{rect}(-1,+1 ; w)(\pi / 4) \sin (\pi|w| / 2) .
\end{aligned}
$$

In our experiments $N$ is equal to $5 \cdot 10^{6}$ in order to obtain a good statistical precision. By using (5) and (11) we obtain the corresponding innovation and the experiment consists in measuring its normalized covariance function. It is clear that the classical conditions of ergodicity of random signals are satisfied, in such a way that the normalized covariance function can be measured by time averaging instead of by ensemble averaging, which is impossible in computer experiment. The results are reported in Table 1 where the different lines correspond to the various forms of PDFs $p_{W}(w)$.

The first line (Bernoulli) corresponds to the simplest case that can be introduced because here $W_{k}$ is a strictly white noise taking only the values \pm 1 with the same probability (Bernoulli white noise).

\begin{tabular}{|c|c|c|c|c|c|c|c|c|c|c|c|}
\hline $\mathrm{PDF} \backslash$ & $p$ & 0 & 1 & 2 & 3 & 4 & 5 & 6 & 7 & 8 & 9 \\
\hline Bernoulli & & 1.000 & 0.000 & 0.000 & 0.000 & 0.001 & 0.000 & 0.000 & 0.001 & 0.000 & 0.000 \\
\hline$p_{1}(w)$ & & 1.000 & 0.000 & 0.000 & 0.001 & 0.000 & 0.000 & 0.001 & 0.000 & 0.000 & 0.000 \\
\hline$p_{2}(w)$ & & 1.000 & 0.000 & 0.000 & 0.000 & 0.000 & 0.000 & 0.000 & 0.000 & 0.000 & -0.001 \\
\hline$p_{3}(w)$ & & 1.000 & 0.000 & 0.000 & 0.000 & 0.000 & 0.001 & 0.000 & 0.000 & 0.000 & 0.000 \\
\hline$p_{4}(w)$ & & 1.000 & 0.000 & 0.000 & -0.001 & 0.000 & 0.000 & -0.001 & 0.000 & 0.000 & 0.000 \\
\hline
\end{tabular}
As a consequence of Property 1, this is also the probability distribution of $X_{k}$, which is then a clipped ARRC(2) signal taking also only the values \pm 1 . The PDFs used in the other four lines are those defined by Eqs. (12) to (15).

Table 1. Covariance function $\gamma_{\tilde{X}}[p]$ of the innovations of some $\operatorname{ARRC}(2)$ signals for various PDFs $p_{W}(w)$.

This table shows clearly that for all the PDFs $p_{W}(w)$ considered the innovation is white with a precision of the order of $10^{-3}$. This means that, according to the Property 2, the normalized covariance functions of all these signals are the same and equal to the one of the classical AR(2) signal (1) defined by the coefficients $a_{1}$ and $a_{2}$. 
The innovation (11) studied in the previous table introduces another interesting consequence for the modeling of the ARRC(2) signal $X_{k}$. Indeed it can be written as $X_{k}=a_{1} X_{k-1}+a_{2} X_{k-2}+\tilde{X}_{k}$, where $\tilde{X}_{k}$ is a sequence of uncorrelated RVs, or a weakly white noise. We have no information about the independence of these RVs, and as a consequence our ARRC(2) signal can also be considered as a weakly $\operatorname{AR}(2)$ signal, as defined above. The marginal PDF $p_{\tilde{X}}(x)$ of the innovation (11) depends only on the parameter of the model, $i$. e. the coefficients $a_{1}, a_{2}$, and the PDF $p_{W}(w)$ of the driving strictly white noise $W_{k}$. Its calculation however is almost impossible and then we shall present below its experimental measurement in the framework of other PDFs measurements.

The innovation (11) is defined, as indicated just before this equation, as the innovation in the linear prediction. It is interesting, however, to note that it is also the innovation in the best mean square prediction. This means simply that this prediction is linear, as it is the case for strictly AR signals. It is well known that the mean square prediction $\hat{X}_{k}$ of $X_{k}$ is the conditional expectation value $\mathrm{E}\left[X_{k} \mid P_{k}\right]$ where $P_{k}$ means the past or the set of RVs $X_{k-j}, j>0$ [1]. It results from (5) and from the definitions of the functions $\psi_{i}($.$) that this expectation is simply a_{1} X_{k-1}+a_{2} X_{k-2}$. We have then the same property as the one of the strictly $\operatorname{AR}(2)$ signals: the mean square prediction is linear and its corresponding innovation is given by (11). This means also that $X_{k}$ is not only weakly but strongly $\operatorname{AR}(2)$, as defined above.

Let us now come to probability distribution analysis. Our purpose is first to verify that, according to Property 1, the marginal DFs of the RVs $X_{k}$ given by (5) are the same as those of the driving noises $W_{k}$, and secondly to determine the PDFs of the innovations $\tilde{X}_{k}$ when the PDFs of $W_{k}$ are those used in covariance measurements reported in the previous table. Each figure corresponds to one of these PDFs. The curves in continuous line represent the PDFs $p_{W}(w)$ of $W_{k}$. The PDFs of $X_{k}$ and $\tilde{X}_{k}$ are measured by normalized histograms and the set of points (.) corresponds to the measurement of the PDFs $p_{X}(x)$ of the RVs $X_{k}$ appearing in (5). The purpose of the experiment is thus to verify that these points are correctly located on the theoretical curve. Finally the sets or points noted (+) correspond to the measurements of the PDFs of the innovation $p_{\tilde{X}}(x)$ for which we have no theoretical result available.

Let us now analyze the marginal PDFs $p_{X}(x)$ of these signals. This is reported in Figures 2 and 3. In these figures we observe that the experimental points (.) are located with a very good precision on the theoretical curves, which means that, according to Property 1, the signals $X_{k}$ have the same marginal distribution as the white noise $W_{k}$. On the other hand we see that, except in Figure 2, the innovation $\tilde{X}_{k}$ defined by (11) has a marginal PDF which does not seem to correspond with any known PDF.

The case of Figure 2 is especially interesting. Indeed the points marked by $(+)$ are located with a very good approximation on the curve $(1 / 2) a \exp (-a|x|)$ with $a=1.3$. But if we use the autoregressive 
modelization (1) where the input signal is a strictly white noise defined by this PDF and with the same values of $a_{1}$ and $a_{2}$ as in all this section, the PDF of the output signal $\bar{X}_{k}$ is no longer a symmetric exponential function. This comes from the fact, already indicated, that the innovation (11) is weakly white, but has no reason to be strictly white.

The clipped ARRC(2) signal corresponding to line 1 of Table 1 cannot be analyzed by the same procedure because it has no PDF since it takes only the values \pm 1 . Its innovation $\tilde{X}_{k}$ defined by (11) is also a discrete valued signal and this equation shows that when the two regression coefficients $a_{i}$ are those chosen previously in the experimental analysis, the only possible values of this innovation are \pm 0.3 , $\pm 0.7, \pm 1.3$, and \pm 1.7 . This appears clearly on Figure 4 where two examples of trajectories of $X_{k}$ and $\tilde{X}_{k}$ are presented. The probabilities $p$ of these values can be calculated and are given in Table 2. Results of experimental measurements $\hat{p}$ of these probabilities are also presented and correspond exactly to the theoretical values.

$\begin{array}{cccccc} & \pm 0.3 & \pm 0.7 & \pm 1.3 & \pm 1.7 & 2 . \text { sum } \\ p & 0.1842 & 0.1842 & 0.0992 & 0.0325 & 1.000 \\ \hat{p} & 0.1842 & 0.1842 & 0.0991 & 0.0325 & 1.000\end{array}$

Table 2. Theoretical $(p)$ and experimental $(\hat{p})$ values of the probabilities of the innovation of the clipped ARRC(2) signal.

Other experiments have been realized with other values of $a_{1}$ and $a_{2}$, including also the cases where the two poles of the TF are real and the results are quite similar. We have then presented various examples of signals $X_{k}$ with arbitrary given marginal PDFs and such that their second-order properties are those of $\operatorname{AR}(2)$ models.

2) Order 4: Let us consider the AR(4) model in which the four reflection coefficients are equal to $k$. By using the Levinson recursion [1], [2] the four regression coefficients are $a_{1}=k-3 k^{2}, a_{2}=$ $k-2 k^{2}+2 k^{3}-k^{4}, a_{3}=k-k^{2}+2 k^{3}$, and $a_{4}=k$. For $k=0.2$, all these coefficients are positive and their sum is 0.5904 . The condition indicated above for the application of our method is then satisfied. Furthermore it is easy to verify that the corresponding dynamical filter has two real and two complex poles given by $z_{1}=0.8356, z_{2}=-0.5957$, and $z_{3}=z_{4}^{*}=-0.07996+j 0.6288$.

The signal $X_{k}$ defined by (10) takes now the form

$$
X_{k}=\sum_{i=1}^{4} \epsilon_{i} \psi_{i}\left(B_{k}\right) X_{k-i}+\psi_{5}\left(B_{k}\right) W_{k},
$$


where the functions $\psi_{i}(x)$ are defined by an obvious extension of those of (4). The innovation is defined as in (11) by

$$
\tilde{X}_{k}=X_{k}-\sum_{i=1}^{4} a_{i} X_{k-i}
$$

The results reported in Table 3 are the same as in Table 1, but correspond to the present ARRC(4) model.

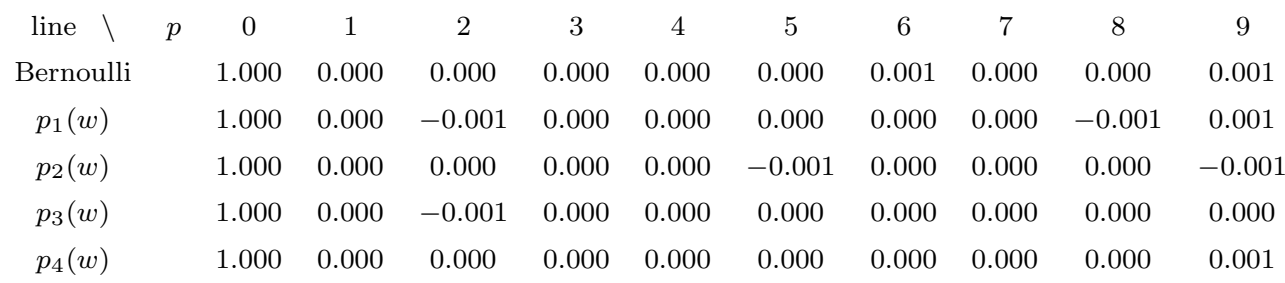

Table 3. Values of the normalized covariance function $\gamma_{\tilde{X}}[p]$ of the innovation of the ARRC(4) signal $X_{k}$ with constant reflection coefficients, $k_{i}=0.2$.

These results are quite similar to those reported in Table 1 and indicate that with a precision of $10^{-3}$ the innovation is a weakly white signal, which means that the covariance function of the ARRC(4) signal (16) is the same as the one of the $\operatorname{AR}(4)$ signal defined by the same reflection or regression coefficients.

Let us now verify the the PDF $p_{X}(x)$ of $X_{k}$ in (16) is the same as the one of the white noise $W_{k}$. In order to simplify we shall present only one set of results corresponding to the PDF (15) used in Figure 3 for the ARRC(2) signal. The results appear in Figure 5 and are quite similar to those of Figure 3: the points (.) corresponding to the measurements of the PDF $p_{X}(x)$ are clearly located on the theoretical curve $p_{W}(w)$. On the other hand the innovation which, according to Table 2 is a weakly white noise, has a PDF represented by (+) which does not correspond to any known form of PDF.

The case of the clipped ARRC(4) signal is especially interesting. We present in Fig. 6 results similar to those appearing in Fig. 4. The trajectories of the signal $X_{k}$ have the same structure of a Bernoulli signal taking only the values \pm 1 . The innovations however are quite different and in the case of an ARRC(4) signal the innovation can take $2^{5}=32$ possible different values equal to $\pm 1-\sum_{i=1}^{4}( \pm) a_{i}$. Unfortunately the calculation of the probabilities of these possible values is much more complicated than for ARRC(2) clipped signals and consequently we cannot present results similar to those appearing in Table 2. Note that the extreme values of the innovation are $\pm\left(1+\sum_{i=1}^{4}\left|a_{i}\right|\right)$, and in the present case this yields \pm 1.5904 , which clearly appears in Fig. 6 . Similarly the smallest positive value of the innovation is 0.4096 , which also appears in Fig 6. It is also interesting to note that the condition $\sum_{i=1}^{4}\left|a_{i}\right|<1$ used in the construction of the model ensures that the values of the innovation $\tilde{X}_{k}$ corresponding to $X_{k}=1$ are all positive. 
There is another AR(4) model which is especially interesting for our discussion. It corresponds to the case where the regression vector a of (1) is such that $a_{i}=0$ for $i \neq 4$ and $a_{4}=-m^{4}$. The polynomial $D(z)$ appearing in the denominator of its TF is thus $D(z)=z^{4}+m^{4}$ and the corresponding four poles are $m \exp [j(2 k+1) \pi / 4]$. The stability condition is then $m<1$ which implies that $\left|a_{4}\right|<1$. This means that there is no other condition for the introduction of our procedure using random coefficients. The equations (16) and (17) defining the ARRC(4) signal and its innovation become now

$$
X_{k}=-\psi\left(B_{k}\right) X_{k-4}+\left[1-\psi\left(B_{k}\right)\right] W_{k},
$$

where $\psi(x)=\operatorname{rect}\left(0, m^{4} ; x\right)$ and

$$
\tilde{X}_{k}=X_{k}+m^{4} X_{k-4}
$$

We shall now present some experimental results concerning this model and we limit our analysis to the case where $W_{k}$ is a symmetric Bernoulli white noise, which leads, as above, to a clipped ARCA(4) signal.

Let us begin by the presentation of results concerning covariance measurements. The normalized covariance function $\gamma_{\bar{X}}[p]$ of the pure AR(4) signal $\bar{X}_{k}$ defined by $\bar{X}_{k}=-m^{4} \bar{X}_{k-4}+W_{k}$, where $W_{k}$ is a white noise of variance equal to 1 , is equal to 0 , except for $p=4 n, n$ integer, where we have $\gamma_{\bar{X}}[4 n]=(-1)^{n} m^{4 n}$. This appears on the first line of Table 4 in which $m=0.8409$, in such a way that $m^{4}=0.5$. In the second line of this table we present results of the measurements of the covariance function of the ARRC(4) signal defined by (18). We observe that the two signals have approximately the same covariance functions ant this is verified with values of $p$ greater that 17 . In the third line of the table we present experimental results of the covariance function of the innovation $\tilde{X}_{k}$ and we see that,

\begin{tabular}{|c|c|c|c|c|c|c|c|c|c|c|c|c|c|c|c|c|c|}
\hline$p$ & 1 & 2 & 3 & 4 & 5 & 6 & 7 & 8 & 9 & 10 & 11 & 12 & 13 & 14 & 15 & 16 & 17 \\
\hline$\gamma_{\bar{X}}[p]$ & 1.000 & 0 & 0 & 0 & -0.500 & 0 & 0 & 0 & 0.250 & 0 & 0 & 0 & -0.125 & 0 & 0 & 0 & 0.062 \\
\hline$\hat{\gamma}_{X}[p]$ & 1.000 & 0.001 & 0.000 & -0.001 & -0.499 & 0.000 & 0.000 & 0.001 & 0.250 & 0.000 & 0.000 & -0.001 & -0.125 & 0.000 & 0.000 & 0.000 & 0.063 \\
\hline$\gamma_{\tilde{X}}[p]$ & 1.000 & -0.001 & 0.001 & 0.001 & 0.000 & 0.000 & 0.000 & 0.000 & 0.000 & -0.001 & 0.001 & 0.000 & 0.000 & 0.000 & 0.000 & 0.000 & 0.000 \\
\hline
\end{tabular}
with the same precision as in the previous experiments, it is a white noise.

Table 4. Normalized covariance functions of $X_{k}$ and of its innovation $\tilde{X}_{k}$.

$$
\text { Theory: } \gamma_{\bar{X}}[p] \text {, Experiment: } \hat{\gamma}_{X}[p] \text { and } \gamma_{\tilde{X}}[p] \text {. }
$$

Its remains to verify that $W_{k}$ and $X_{k}$ have the same distribution function. It is obvious from (18) that if $X_{1}= \pm 1$, then all the $X_{k}$ s with $k>1$ take the same values \pm 1 . We have verified this point and this appears in Fig. 7 which reports the same kind of results as in Fig. 6 but with the signal given by (18) where $m^{4}=0.5$, as in the experiments of Table 4. It is clear on the trajectory of $X_{k}$ presented in this 
figure that $X_{k}$ is a clipped signal taking only the values \pm 1 . To verify that its DF is the same that the one of $W_{k}$ it is sufficient to verify that these signals have the same mean value 0 . In various experiments with $10^{7}$ samples of $X_{k}$ and $W_{k}$ we have verified that their corresponding mean values are of the order of $10^{-5}$.

On the same figure we present an example of the trajectory of the innovation (19). It is clear from this equation that $\tilde{X}_{k}$ take only the four values $\pm 1 \pm m^{4}$, and this appears on the figure corresponding to an experiment where $m^{4}=0.5$. Let $p_{-}$and $p_{+}$be the probabilities that $\tilde{X}_{k}$ takes the values $1-m^{4}$ and $1+m^{4}$ respectively. The values of these probabilities can be calculated and the results are compared to those given by experiments reported in Table 5. We present these values for various values of the parameter $m$, modulus of the poles of the filter generating the signal $X_{k}$. The experimental results are noted $\hat{p}_{-}$and $\hat{p}_{+}$and we see that they correspond with an excellent approximation to their theoretical values. The column $S$ corresponds to the sum of the results of the previous two lines.

$\begin{array}{cccccccc}m & m^{4} & p_{-} & p_{+} & S & \hat{p}_{-} & \hat{p}_{+} & \hat{S} \\ 0.5623 & 0.1000 & 0.2750 & 0.2250 & 0.5000 & 0.2751 & 0.2248 & 0.4999 \\ 0.7401 & 0.3000 & 0.3250 & 0.1750 & 0.5000 & 0.3250 & 0.1752 & 0.5002 \\ 0.8409 & 0.5000 & 0.3750 & 0.1250 & 0.5000 & 0.3749 & 0.1251 & 0.5000 \\ 0.9147 & 0.7000 & 0.4250 & 0.0750 & 0.5000 & 0.4251 & 0.0750 & 0.5001 \\ 0.9740 & 0.9000 & 0.4750 & 0.0250 & 0.5000 & 0.4750 & 0.0250 & 0.5000 \\ 0.9873 & 0.9500 & 0.4875 & 0.0125 & 0.5000 & 0.4875 & 0.0125 & 0.5000\end{array}$

Table 5. Probabilities of the innovations for various values of $m$.

Theory: $p_{-}$and $p_{+}$. Experiment: $\hat{p}_{-}$and $\hat{p}_{+}$.

\section{MARC MODELS}

\section{A. Review on MA Models}

We shall now extend the previous ideas to the case of moving average (MA) signals. A MA( $m$ ) signal $\bar{X}_{k}$ is defined by the relation

$$
\bar{X}_{k}=\sum_{i=0}^{m} b_{i} W_{k-i}
$$

where, as previously, $W_{k}$ is a strictly white noise. The assumption of weak whiteness is sufficient if one is only interested in the second-order properties of $\bar{X}_{k}$. This relation exhibits the input-output relationship of a finite impulse response (FIR) filter and the coefficients $b_{i}$ are the values of its impulse response (IR). These coefficients are finite and since their number is finite (FIR), there is never a stability problem, unlike the case of autoregressive signals. When the input is strictly white, each RV $\bar{X}_{k}$ is a sum of $m+1$ independent RVs and the PDF of $\bar{X}_{k}$ can be obtained by a multiple convolution. The calculation, however, 
is often very complicated and the explicit expression of this PDF can rarely be obtained in closed form, except in the important case of Gaussian input white noise. On the other hand the covariance function of $\bar{X}_{k}$ is given by the very simple expression

$$
\gamma_{p}=\left(b_{0} b_{|p|}+b_{1} b_{|p|+1}+\ldots+b_{m-|p|} b_{m}\right) \sigma_{W}^{2}, 0 \leq|p| \leq m,
$$

where $\sigma_{W}^{2}$ is the variance of $W_{k}$, and $\gamma_{p}=0$ if $|p|>m$.

\section{B. FIR Filters with Random Coefficients}

As in the section on ARC signals we shall compare classical FIR filters and FIR filters with random coefficients (FIRRC). The input-output relationship in classical causal FIR filters is the convolution (20) where the input and output are now written $X_{k}$ and $\bar{Y}_{k}$ respectively.

For the introduction of FIRRC filters let us first introduce the coefficients $\epsilon_{i}$ defined as in (2) by

$$
\epsilon_{i}=b_{i} /\left|b_{i}\right|=\operatorname{sign}\left(b_{i}\right) ; 0 \leq i \leq m .
$$

Let also $S$ be the sum

$$
S=\sum_{i=0}^{m}\left|b_{i}\right|=\sum_{i=0}^{m} \epsilon_{i} b_{i}
$$

and $\pi_{i}$ be the quantity

$$
\pi_{i}=\frac{\left|b_{i}\right|}{S}=\frac{\epsilon_{i} b_{i}}{S} .
$$

It results from (23) that $\sum_{i=0}^{m} \pi_{i}=1$. With these numbers we make a partition of the interval $[0,1]$ into $m+1$ intervals defined as follows. Let $s_{i}$ be defined by the recursion $s_{i}=s_{i-1}+\pi_{i}, 1 \leq i \leq m$, with the initial condition $s_{0}=\pi_{0}$. As a consequence of (23) and (24), we have $s_{m}=1$. The first function $\psi_{i}(x)$ is $\psi_{0}(x)=\operatorname{rect}\left(0, s_{0} ; x\right)$, and the others for $1 \leq i \leq m$ are

$$
\psi_{i}(x)=\operatorname{rect}\left(s_{i-1}, s_{i} ; x\right) .
$$

Let, as previously, $B_{k}$ be a strictly white noise with an uniform distribution in $[0,1]$. The FIRRC inputoutput relationship is then defined by

$$
Y_{k}=\sum_{i=0}^{m} \psi_{i}\left(B_{k}\right) \epsilon_{i} X_{k-i} .
$$

Property 3. If the marginal probability distribution of $X_{k}$ is symmetric, then $X_{k}$ and $Y_{k}$ defined by (26) have the same marginal probability distribution.

Proof. Equation (26) means that $X_{k}$ is equal to $\epsilon_{i} X_{k-i}$ with the probability $\pi_{i}$ given by (24). This allows us to calculate the characteristic function of $X_{k}$. By using again the assumption of symmetry of 
the PDF $p_{X}(x)$ of $X_{k}$, we deduce that the PDF of $\epsilon_{i} X_{k-i}$ is $p_{X}(x)$, and, as a result, this PDF is also the marginal PDF of $Y_{k}$.

Property 4. If $\bar{Y}_{k}$ and $Y_{k}$ are obtained from $X_{k}$ by a convolution like (20) and by (26) respectively, then their covariance functions $\gamma_{Y}[p]$ and $\gamma_{\bar{Y}}[p], p \neq 0$, are proportional.

Proof. The covariance function $\gamma_{\bar{Y}}$ of $\bar{Y}_{k}$ given by (20) when the input is $X_{k}$ instead of $W_{k}$ is given by the classical expression

$$
\gamma_{\bar{Y}}[p]=\sum_{i=0}^{m} \sum_{j=0}^{m} b_{i} b_{j} \gamma_{X}[p-i+j]
$$

where $\gamma_{X}[p]$ is the covariance function of $X_{k}$. This yields (21) when $X_{k}$ is white.

Consider now the case of $Y_{k}$ given by (26). For $p=0$ we deduce from the relations $\psi_{i}(x) \psi_{j}(x)=0$ and $\psi_{i}^{2}(x)=\psi_{i}(x)$, already used in Section 2 , that

$$
Y_{k}^{2}=\sum_{i=0}^{m} \psi_{i}\left(B_{k}\right) X_{k-i}^{2}
$$

and since $\mathrm{E}\left[\psi_{i}\left(B_{k}\right)\right]=\pi_{i}$, we deduce that $\gamma_{Y}[0]=\gamma_{X}[0]$, which is also a consequence of Property 3 .

Consider now the case $p \neq 0$. Since $B_{k}$ is strictly white and independent of $X_{k}$, we can write

$$
\gamma_{Y}[p]=\sum_{i=0}^{m} \sum_{j=0}^{m} \epsilon_{i} \epsilon_{j} \mathrm{E}\left[\psi_{i}\left(B_{k}\right) \psi_{j}\left(B_{k-p}\right)\right] \mathrm{E}\left[X_{k-i} X_{k-p-j}\right]=\sum_{i=0}^{m} \sum_{j=0}^{m} \epsilon_{i} \epsilon_{j} \pi_{i} \pi_{j} \gamma_{X}[p+j-i],
$$

and by using (24) and (27), we deduce that $\gamma_{Y}[p]=(1 / S) \gamma_{\bar{Y}}[p]$, which is Property 4.

This can be summarized in only one expression written as

$$
\gamma_{Y}[p]=(1 / S) \gamma_{\bar{Y}}[p]+\delta[p]\left\{\gamma_{X}[0]-(1 / S) \gamma_{\bar{Y}}[0]\right\}
$$

where $\delta[p]$ is the Kronecker-delta signal equal to 1 for $p=0$ and to 0 otherwise.

We shall now apply these general expressions to introduce MARC and ARMARC signals.

\section{MARC(m) Signals}

Let $\bar{X}_{k}$ be the MA $(m)$ signal defined by (20). With the same values of $m$ and $b_{i}$ we can construct the random signal defined by (26) in which the input is now $W_{k}$ and the output is called $X_{k}$. This signal $X_{k}$ is a MARC $(m)$ signal and it results from Properties 3 and 4 that it has the same marginal PDF as the driving white noise $W_{k}$ and also that the values of its covariance function for $p \neq 0$ are proportional to those of $\bar{X}_{k}$.

As for ARRC signals we shall now verify these results in experiments with simulated MARC signals.

These experiments are realized with a MARC(10) signal in which the coefficients are $b_{i}=1$ for $0 \leq i \leq 4$ and $b_{i}=-1$ for $5 \leq i \leq 9$. Results of PDF measurements are presented in Fig. 8 obtained 
when the PDFs of $W_{k}$ are given by (12) or (15). As in the previous figures the continuous lines represent these two functions and the points are the results of the measurements of the PDFs of $X_{k}$ in an experiment using $5 \cdot 10^{6}$ values of this signal. We observe that these points are perfectly localized on the continuous curves, which shows that the measured PDFs of $X_{k}$ are equal to those of the driving noise $W_{k}$.

In Figure 9 we consider the case where $W_{k}$ is a symmetric Bernoulli white noise. As indicated above it is not possible here to make an analysis with PDF measurements, because this PDF does not exist. The signal $X_{k}$ itself, however, can be represented and we see in the figure that it is also a Bernoulli signal, but with the covariance of an MA(10) signal, which is verified below. On the other hand we have also presented in the figure the signal $Y_{k}$ equal to $\bar{X}_{k}$ given by (20), where the coefficients $b_{i}$ and the driving noise $W_{k}$ are the same as for $X_{k}$. As verified below the two signals have the same covariance functions, but it is clear that their structures are quite different.

Let us now present results of covariance measurements realized with the MARC(10) signal defined by the same coefficients $b_{i}$ and the same PDFs $p_{W}(w)$ as in the PDFs measurements. This appears in the following Table 6 where values of covariance function measurements for $1 \leq p \leq 10$ are presented, because the value for $p=0$ requires a specific treatment. As these values must be proportional we have normalized, the results in such a way that these values become the same.

The results of this table require the following comments. In line 1 we present the values of $\gamma_{p}$ given by (21) for an input white noise $W_{k}$ of unit variance. This covariance decreases by steps of amplitude 3 for $p$ from 0 to 5 , and afterwards increases by steps of amplitude 1 for $p$ from 6 to 10. It is zero for $|p| \geq 10$. In line 2 we present experimental measurements of the covariance function $\gamma_{p}$ of a simulated Gaussian pure MA(10) signal. We observe that they coincide almost perfectly with the theoretical values of the previous line. The following lines are devoted to various MARRC(10) signals. Their coefficients $b_{i}$ are the same as in lines 1 and 2 and their difference comes uniquely from the $\operatorname{PDF} p_{W}(w)$ of the driving noise $W_{k}$. These PDFs are those given by Eqs. (12) to (15). In the last line the results correspond to the case where the input signal $W_{k}$ is a symmetric Bernoulli white noise. We observe that the general behavior of the experimental results correspond correctly with the theoretical calculations. The precision, however, is not so accurate as in the case of autoregressive signals. 


$\begin{array}{cccccccccccc} & p & 1 & 2 & 3 & 4 & 5 & 6 & 7 & 8 & 9 & 10 \\ \text { MA(10), Theory, Eq. (21) } & 7 & 4 & 1 & -2 & -5 & -4 & -3 & -2 & -1 & 0 \\ \text { MA(10) Gaussian } & 7.00 & 4.00 & 1.00 & -2.00 & -5.00 & -4.00 & -3.00 & -2.01 & -1.00 & 0.00 \\ \text { MARRC(10), p } 1(w) & 7.06 & 3.98 & 0.97 & -1.95 & -4.92 & -4.02 & -2.95 & -1.99 & -1.03 & 0.00 \\ \text { MARRC(10), p } 4(w) & 7.00 & 3.99 & 1.03 & -2.01 & -4.92 & -4.07 & -3.06 & -2.00 & -1.00 & 0.02 \\ \text { MARRC(10), p } 2(w) & 7.02 & 4.03 & 1.02 & -2.02 & -5.01 & -3.99 & -3.00 & -2.05 & -0.93 & -0.06 \\ \text { MARRC(10), Bernoulli } & 7.01 & 4.03 & 1.03 & -1.92 & -4.91 & -3.96 & -3.02 & -2.03 & -1.02 & -0.05\end{array}$

Table 6. Covariance functions of MA (10) and MARC(10) signals.

\section{ARMARC MODELS}

Consider an $\operatorname{AR}(r)$ signal $\bar{X}_{k}$ defined by (1) and the signal $\bar{Y}_{k}$ defined by (20) where the input $W_{k}$ is replaced by $\bar{X}_{k}$ and the output is noted $\bar{Y}_{k}$. This signal is called an ARMA $(r, m)$ model. Note that, since the input-output relationships in these two filters are convolutions and since the product of convolutions is commutative, the order in the sequence AR MA or MA AR does not play any role. The parameters defining the covariance properties of an ARMA signals are then the $r$ coefficients $a_{i}$ of the AR part and the $m$ coefficients $b_{i}$ of the MA part.

Consider now the signal $Y_{k}$ defined from the strictly white noise $W_{k}$ by

$$
Y_{k}=\sum_{i=0}^{m} \Psi_{i}\left(N_{k}\right) \eta_{i} X_{k-i} ; X_{k}=\sum_{i=1}^{r} \psi_{i}\left(B_{k}\right) \epsilon_{i} X_{k-i}+\psi_{r+1}\left(B_{k}\right) W_{k},
$$

in which $B_{k}$ and $N_{k}$ are two strictly white and independent signals with a marginal distribution uniform in $[0,1]$. The functions $\psi_{i}(x)$ and the coefficients $\epsilon_{i}$ are defined as in (10), in such a way that $X_{k}$ is the ARRC signal defined by (10) and studied in Section II C. On the other hand the parameters $\eta_{i}$ and the functions $\Psi_{i}(x)$ are those appearing in (26) and called $\epsilon_{i}$ and $\psi_{i}(x)$ respectively and defined from the parameters $b_{i}$, in such a way that $Y_{k}$ is obtained from $X_{k}$ by a FIRRC, as described in Section III B.

It results from the previous analysis that $Y_{k}$ and $W_{k}$ have the same marginal PDF. On the other hand the normalized covariance function of $X_{k}$ is the same as the one of the $\operatorname{AR}(r)$ signal $\bar{X}_{k}$ defined by the coefficients $a_{i}$ appearing in (1) and it results from Property 4 that the covariance functions $\gamma_{Y}[p]$ and $\gamma_{\bar{Y}}[p]$ are proportional for $p \neq 0$. As $\gamma_{\bar{Y}}[p]$ is the covariance function of an ARMA $(r, m)$ signal defined by the $a_{i}$ s and the $b_{i}$ s, we have then constructed an ARMARC $(r, m)$ signal with an arbitrary marginal PDF and with the covariance properties of a pure ARMA $(r, m)$ signal, which was the purpose of this paper.

Note finally that the two operations appearing in (31) are not commutative because the strictly white noise $W_{k}$ must be applied to construct the AR part of the signal. 
It remains to verify these results in computer experiments. For this purpose we start from the ARMA $(4,4)$ signal in which the coefficients of the AR part are $a_{1}=a_{2}=a_{3}=0, a_{4}=-m^{4}$, while those of the MA part are $b_{i}=(-1)^{i}, 0 \leq i \leq 3$. The signal $X_{k}$ is then the one described by (18).

We do not present results of PDF measurements because they are completely similar to those obtained in the previous section: whatever the PDF $p_{W}(w)$ of the driving noise $W_{k}$, the experimental points of the measurements of the PDF of $Y_{k}$ show clearly that $Y_{k}$ and $W_{k}$ have the same PDF.

As the theoretical expressions of the covariance functions of $Y_{k}$ are rather complicated, the purpose of our experiments is to show that the covariance functions of $\bar{Y}_{k}$ and $Y_{k}$ are the same after convenient normalisation for various values of the parameter $m$. The principle of the normalisation starts from the point noted above that the values of the covariance functions $\gamma[p]$ for $p=0$ are insignificant and must be eliminated. Then the signals $\bar{Y}_{k}$ and $Y_{k}$ are normalized in such a way that the values of their covariance functions for $p=1$ are the same, and the purpose of the experiment is to verify that, as a result, all the other values are approximately equal. This appears on the Table 7.

$\begin{array}{cccccccccccc} & p & 1 & 2 & 3 & 4 & 5 & 6 & 7 & 8 & 9 & 10 \\ m=0 & \gamma_{\bar{Y}}[p] & -0.750 & 0.501 & -0.251 & 0.002 & -0.001 & 0.001 & -0.001 & 0.000 & -0.000 & -0.000 \\ & \gamma_{Y}[p] & -0.750 & 0.504 & -0.251 & 0.002 & -0.001 & 0.001 & 0.000 & 0.001 & 0.002 & -0.002 \\ m=0.3 & \gamma_{\bar{Y}}[p] & -0.675 & 0.350 & -0.024 & -0.300 & 0.203 & -0.106 & 0.008 & 0.089 & -0.060 & 0.031 \\ & \gamma_{Y}[p] & -0.675 & 0.350 & -0.028 & -0.302 & 0.204 & -0.108 & 0.007 & 0.089 & -0.060 & 0.030 \\ m=0.5 & \gamma_{\bar{Y}}[p] & -0.625 & 0.250 & 0.125 & -0.500 & 0.313 & -0.126 & -0.062 & 0.250 & -0.157 & 0.063 \\ & \gamma_{Y}[p] & -0.625 & 0.249 & 0.124 & -0.500 & 0.315 & -0.124 & -0.060 & 0.249 & -0.156 & 0.065 \\ m=0.7 & \gamma_{\bar{Y}}[p] & -0.575 & 0.150 & 0.275 & -0.699 & 0.403 & -0.105 & -0.192 & 0.489 & -0.282 & 0.073 \\ & \gamma_{Y}[p] & -0.575 & 0.150 & 0.276 & -0.701 & 0.404 & -0.106 & -0.192 & 0.489 & -0.283 & 0.072\end{array}$

Table 7. First ten values of the covariance functions $\gamma_{\bar{Y}}[p]$ and $\gamma_{Y}[p]$ for various values of $m$.

Note that for $m=0, X_{k}=W_{k}$, and $Y_{k}$ becomes a MARC (4) signal. We see effectively in the first two lines of the table that the covariance functions are approximately 0 for $p \geq 4$, which characterizes a MA(4) model. Furthermore the numerical values of the covariance function are those corresponding to the coefficients $b_{i}$ chosen. For the other values of $m$ we observe that, as expected from the theoretical analysis, the covariance functions of the ARMA and ARMARC are practically the same.

\section{Conclusion}

$\operatorname{AR}(r), \operatorname{MA}(m)$ and $\operatorname{ARMA}(r, m)$ signals are of common use in many fields of Signal Processing. Their second-order properties (covariance functions and power spectra) are very well investigated. On the other hand, except in the Gaussian case, it is very difficult, and sometimes even impossible, to obtain 
the structure of their probability distribution, except for small values of the order $r$ or $m$. The purpose of this paper was to overcome a part of this difficulty.

By using a structure with appropriate random coefficients we have shown that it is possible to construct signals with ARMA second-order properties, while the marginal PDF is arbitrary but symmetric and given in advance. Computer experiments in which covariance functions and PDFs are measured confirm completely the results of the theoretical analysis of such models.

However various open problems remain. It would be interesting to calculate multivariate probability distributions of such models because in some problems of statistical Signal Processing the marginal distribution is insufficient. Some results on this problem are already obtained and ready for another publication. Finally it would be of interest to suppress some assumptions introduced in the calculations, as for example the symmetry of the PDFs or some constraints on the coefficients of the autoregressive part of the models.

\section{REFERENCES}

[1] Picinbono B., Random Signals and Systems, Englewood Cliffs, N.J. : Prentice Hall, 1993.

[2] Kay S., Modern Spectral Estimation, Englewood Cliffs, N.J. : Prentice Hall, 1988.

[3] Liu B., Munson D., "Generation of a random sequence having a jointly specified marginal distribution and autocovariance," IEEE Trans. on Acoustics, Speech and Signal processing, vol. ASSP 30, pp. 973-983, December 1982.

[4] Sondhi M.M., "Random processes with specified spectral density and first-order probability density," Bell System Technical Journal, vol. 62, pp. 679-702, 1983.

[5] Conte E., Lops M., Ricci G., "Generation of random sequences with prescribed marginal distribution and autocovariance function," International Symposium on Communication Theory and its Applications, Warwick (Scotland), September 1991.

[6] Nicholls D.F. and Quinn B.G., Random Coefficient Autoregressive Models: an Introduction, Lectures Notes in Statistics, 11, New-York-Berlin : Springer-Verlag, 1982.

[7] Boshnakov G.N., "On first and second order stationarity of random coefficient models," Research report No. 3, School of matehmatics, The University of Manchester, http://www.maths.manchester.ac.uk/ gb/Publications/publications.html, 2009.

[8] Granger C.W.J. and Anderson A.P., An introduction to bilinear times series analysis, Göttingen : Vanenehoeck and Rupecht, 1978.

[9] Andel J., "Autoregressive series with random parameters," IEEE Math. Operationfors. Stat. Statist. Optim, 7, pp. 735-741, 1976.

[10] Quinn B.G. and Nicholls D.F., "The stability or random coefficients autoeregressive models," Int. Econ. Rev., vol. 22, pp. 741-744, December 1982. 
Bernard Picinbono (M'63-SM'79-F'85) was born in Algiers, Algeria, on March 21, 1933. He received the Agrgation des Sciences Physiques degree in 1956 and the Doctorat d'État degree in 1960. From 1960 to 1965, he was a Professor with the Department of Physics, University of Algiers, and in 1965, he joined the University of Paris-Sud, Orsay, France, to which he is still attached (Emeritus). He was the President of this university between 1971 and 1975 and Director of the Laboratoire des Signaux et Systèmes (L25), which is a joint research center of the Centre National de Ia Recherche Scientifique (CNRS), the École Suprieure d'Électricité (ESE), and the Université de Paris-Sud (UPS) between 1975 and 1987. Between 1990 and 1995, he was General Director of the ESE. His research interests include statistical signal processing, detection and estimation, and stochastic processes. He is the author of numerous papers and books on these subjects. Dr. Picinbono is a member of the French Academy of Sciences and of the Académie des Technologies. He was President of the GRETSI Conference organized every two years in France and devoted to Signal and Image Processing problems. He was Co-Chairman of the 1982 IEEE International Symposium of Information Theory, Les Arcs, France. 


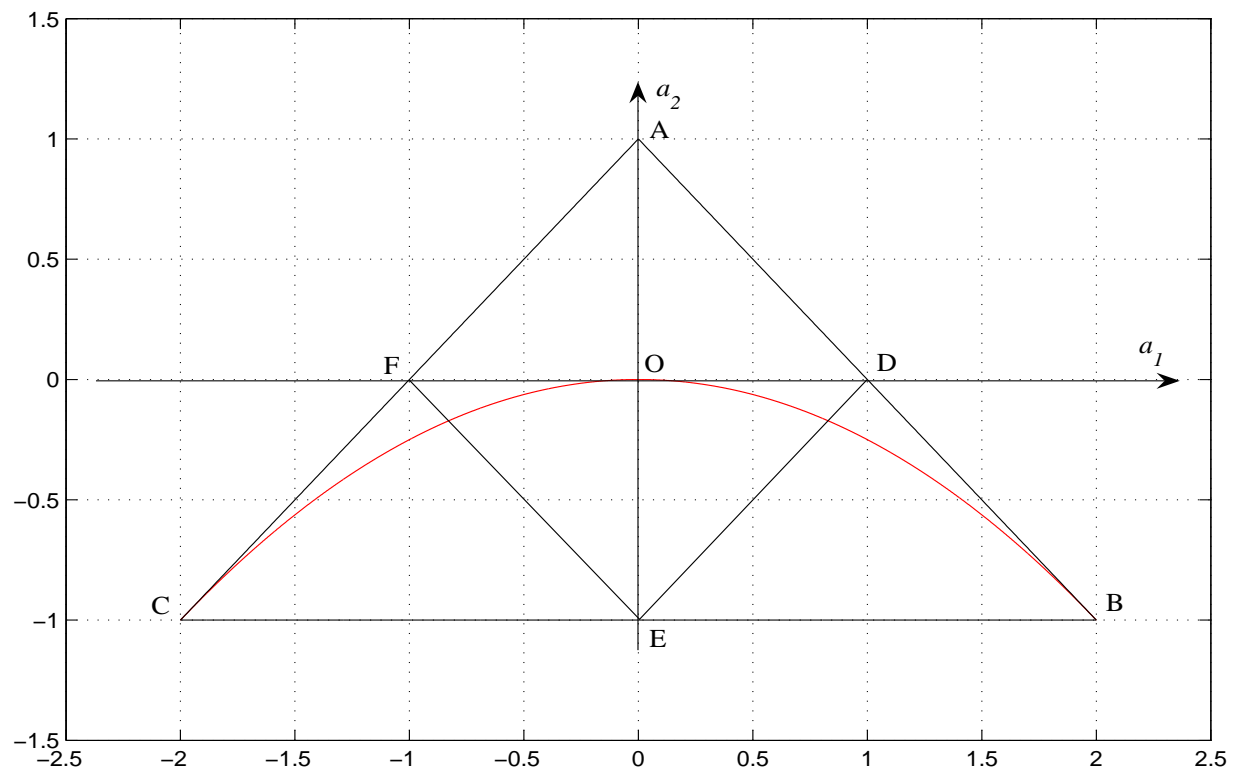

Fig. 1. Triangle of stability of an AR(2) model. 


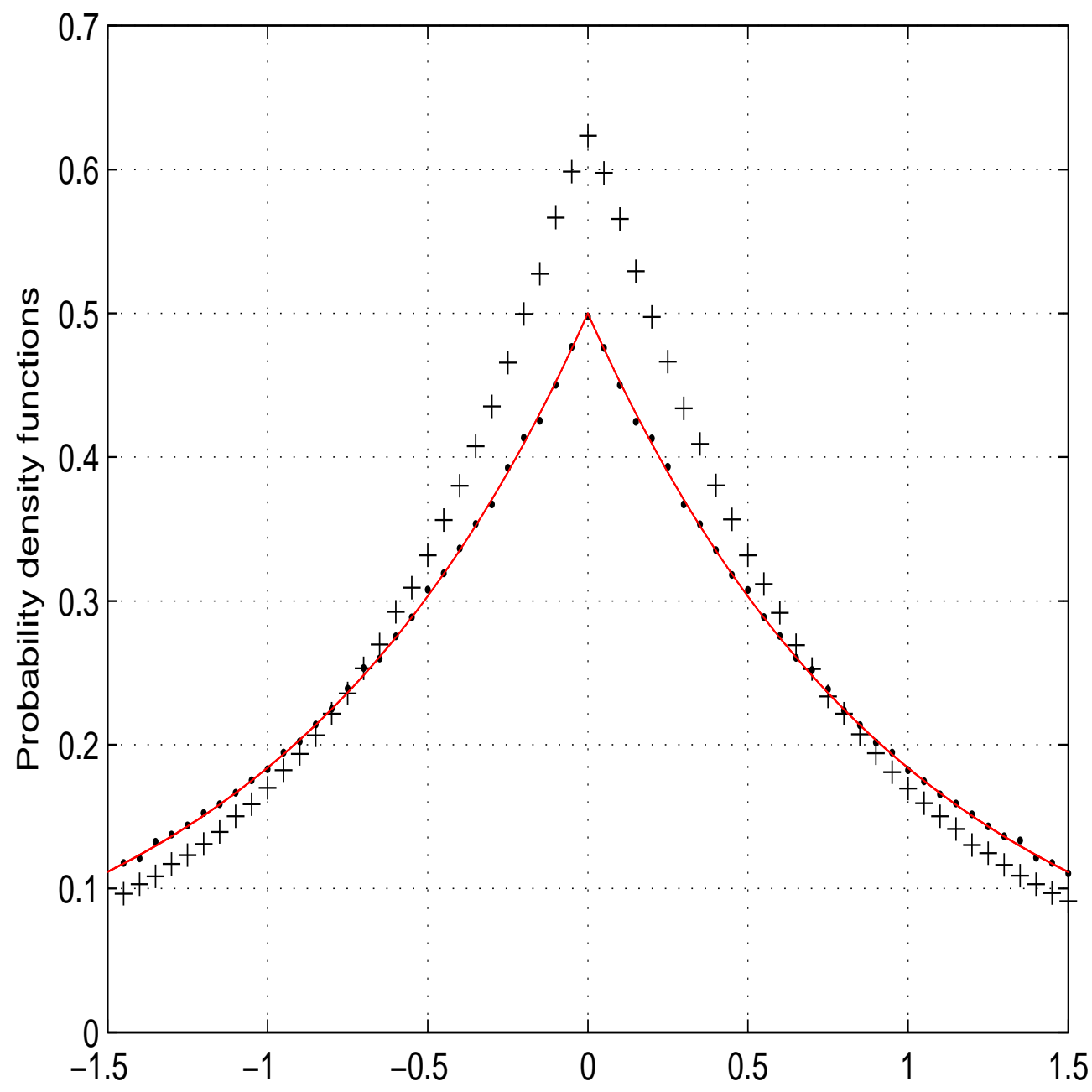

Fig. 2. PDFs $p_{X}(x)($.$) and p_{\tilde{X}}(x)(+)$ of the ARRC(2) signal with a symmetric exponential distribution $p_{1}(w)$ defined by (12) (continuous line) for $a_{1}=0.2$ and $a_{2}=-0.5$. 


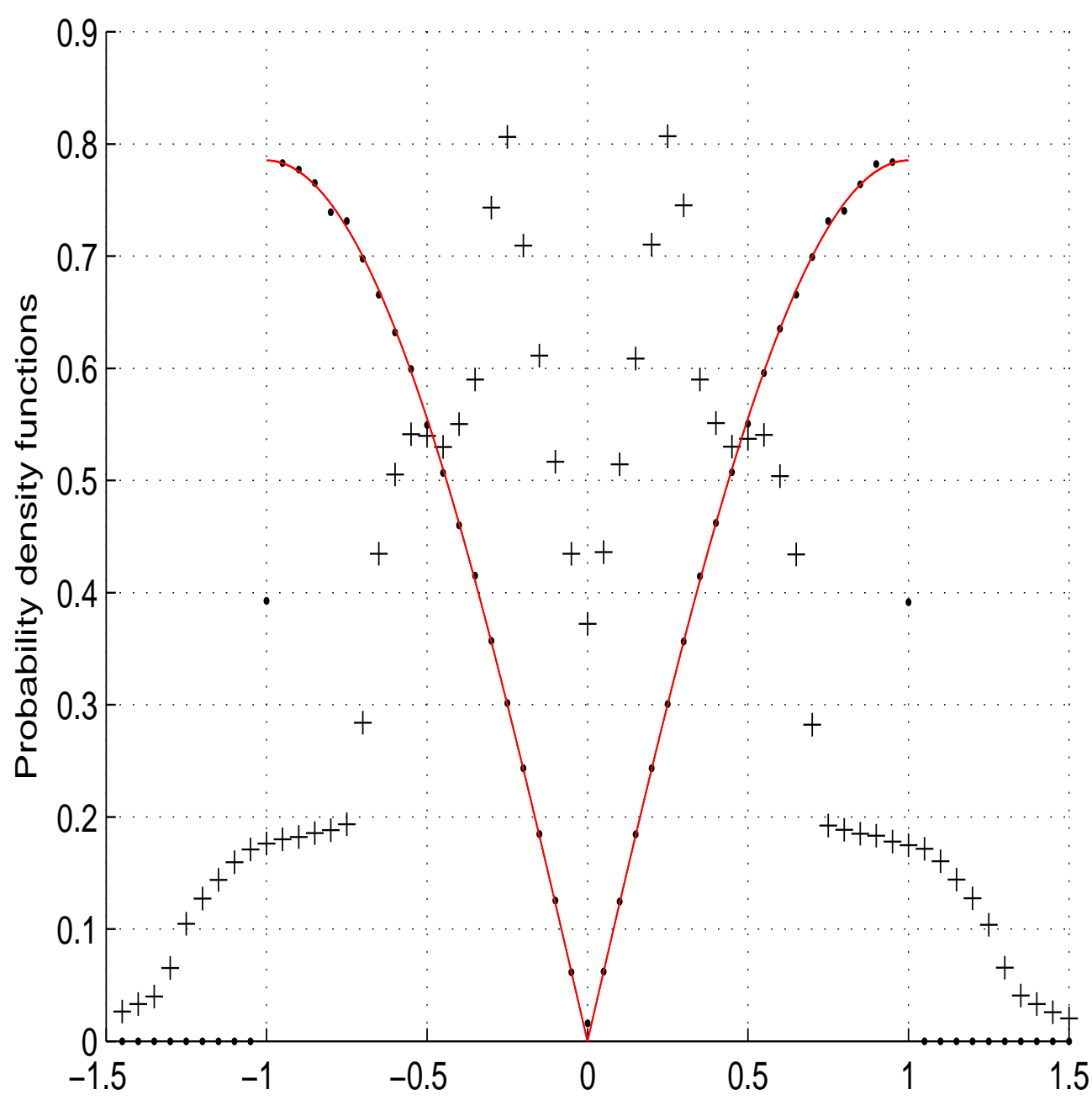

Fig. 3. PDFs $p_{X}(x)($.$) and p_{\tilde{X}}(x)(+)$ of the ARRC(2) signal with the distribution $p_{4}(w)$ defined by (15) (continuous line) for $a_{1}=0.2$ and $a_{2}=-0.5$. 

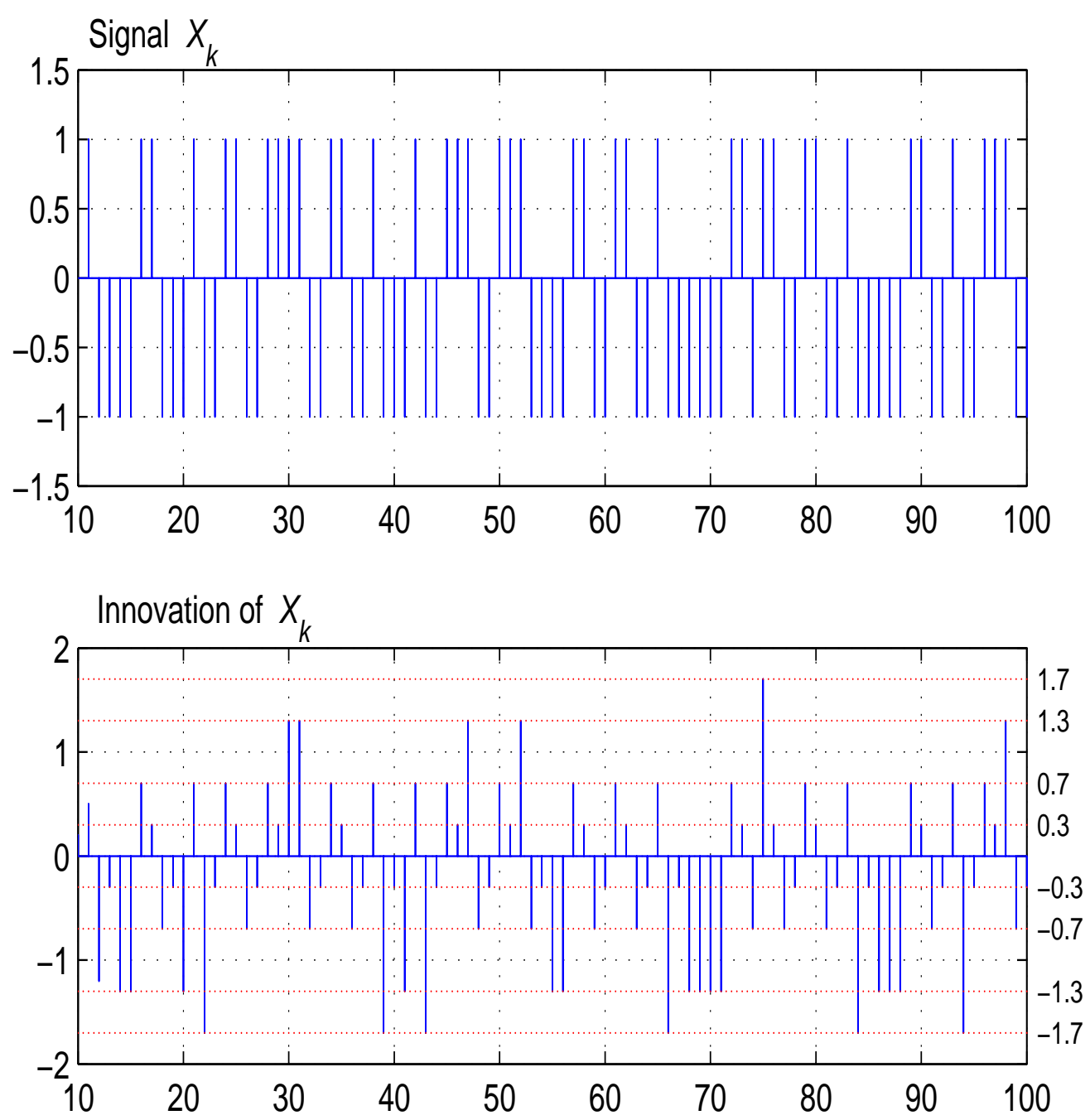

Fig. 4. Clipped ARRC(2) signal $X_{k}$ and its innovation $\tilde{X}_{k}$ for $a_{1}=0.2$ and $a_{2}=-0.5$. 


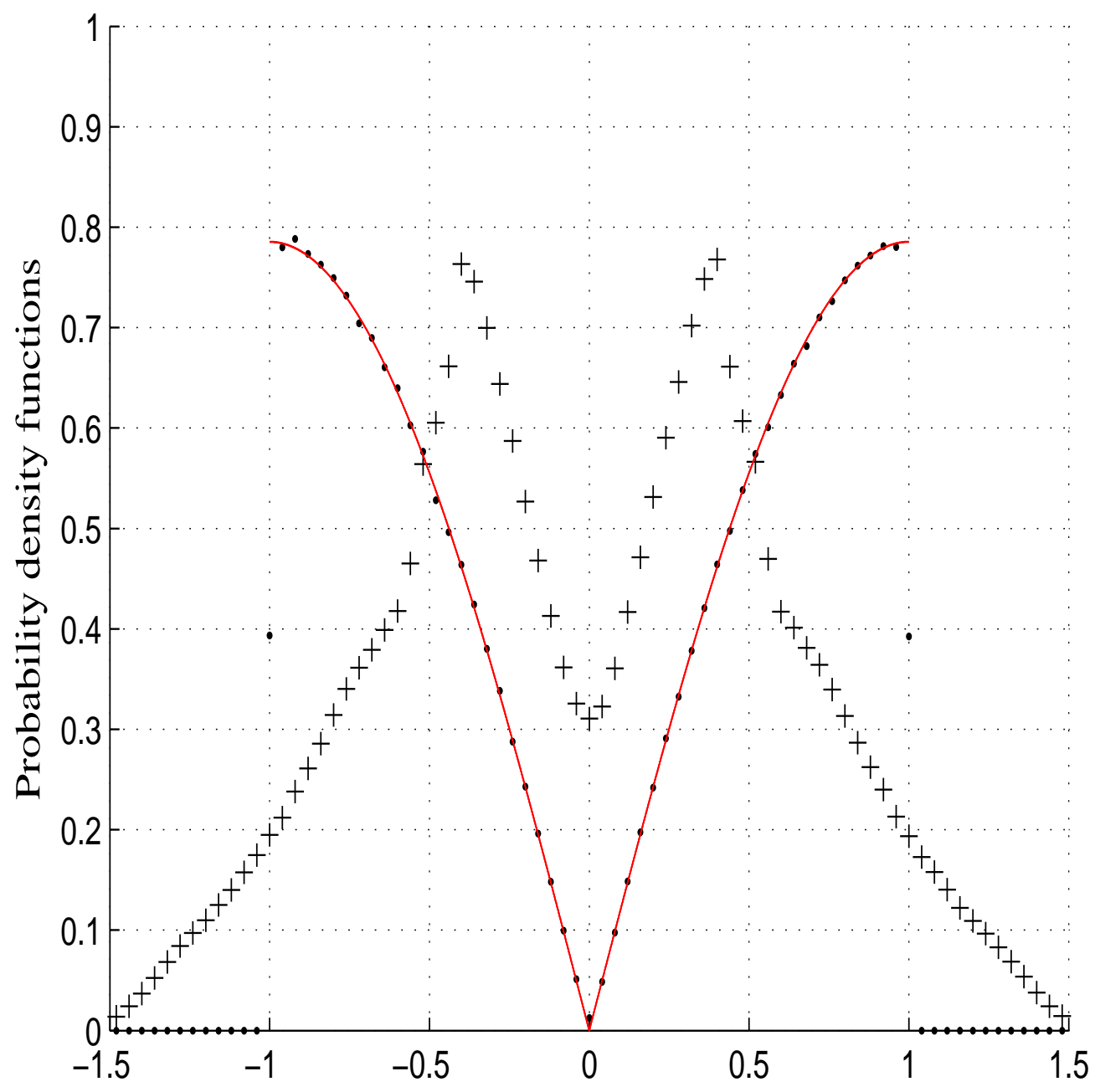

Fig. 5. PDFs $p_{X}(x)($.$) and p_{\tilde{X}}(x)(+)$ of the ARRC(4) signal with constant reflection coefficients and for the distribution $p_{4}(w)$ defined by (15) (continuous line). Reflection coefficients $k_{i}=0.2$. 

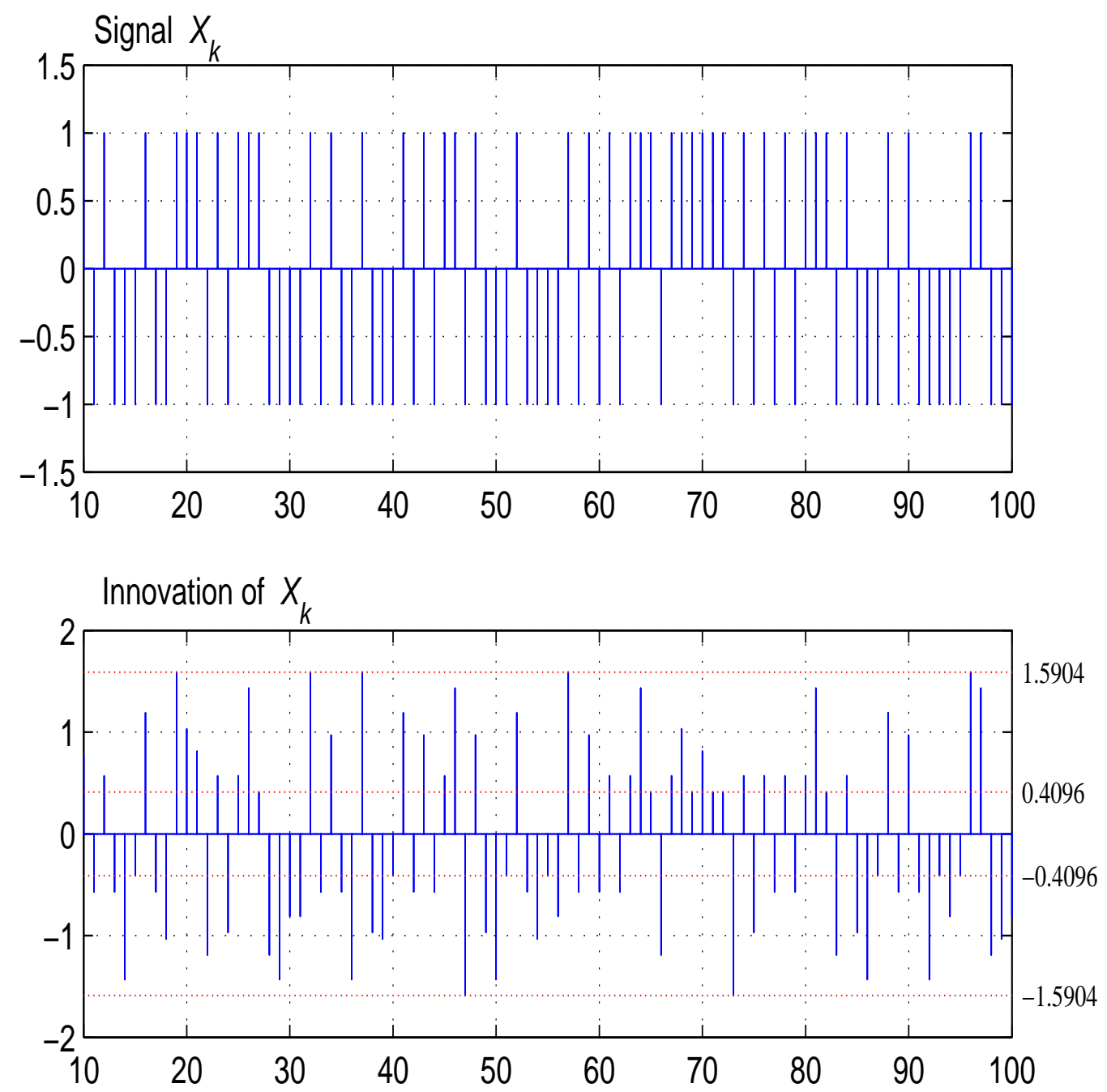

Fig. 6. Clipped ARRC(4) signal $X_{k}$ with constant reflection coefficients and its innovation $\tilde{X}_{k}$ for $k_{i}=0.2$. 

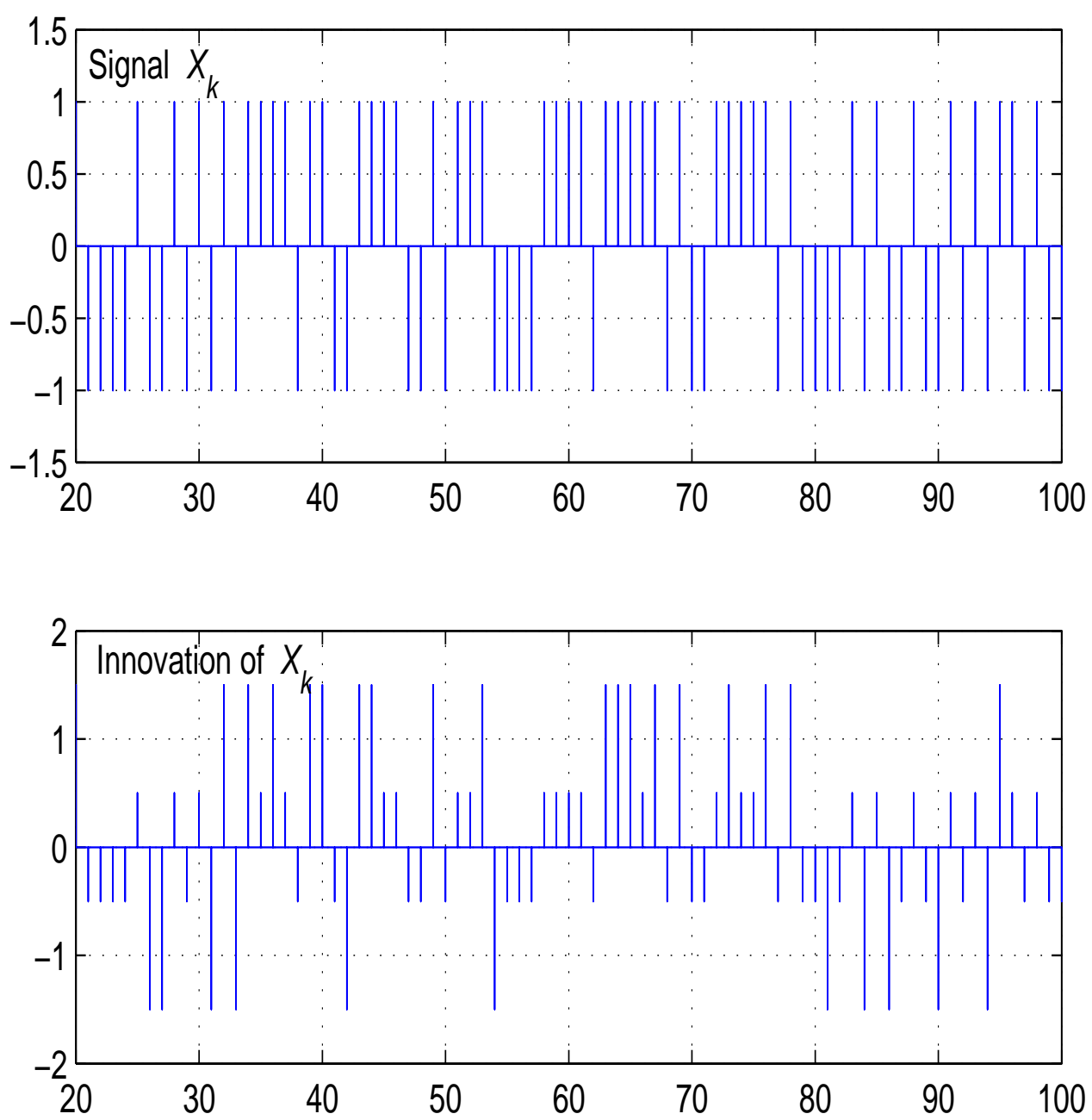

Fig. 7. Clipped ARRC(4) signal $X_{k}$ of (18) and its innovation $\tilde{X}_{k}$ of (19) for $m^{4}=0.5$. 


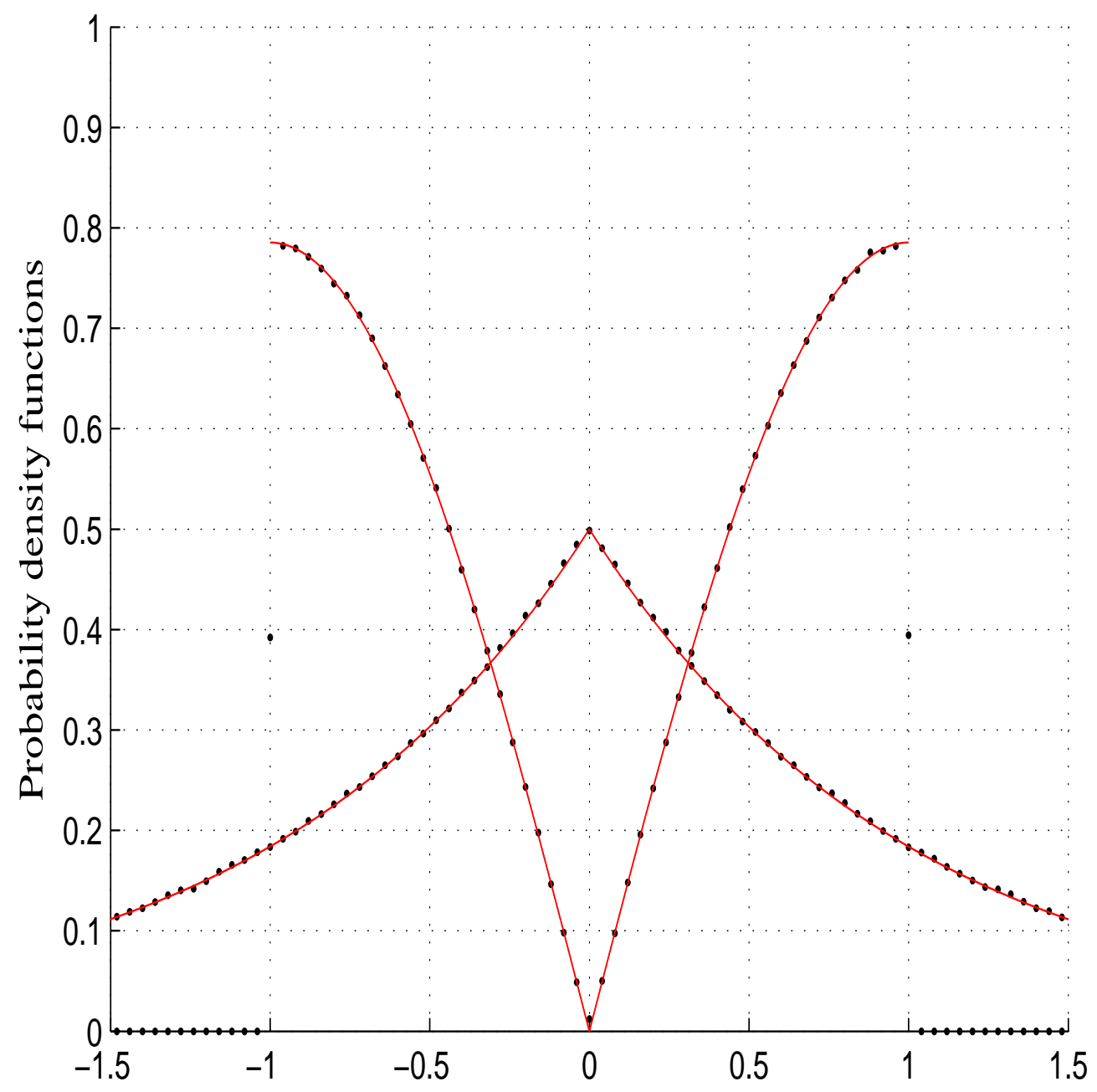

Fig. 8. PDFs $p_{X}(x)$ (.) of the MARC(10) signal with $p_{W}(w)=p_{1}(w)$ of (12) and $p_{4}(w)$ of (15). Points : experiment. Continuous curve : theory. 

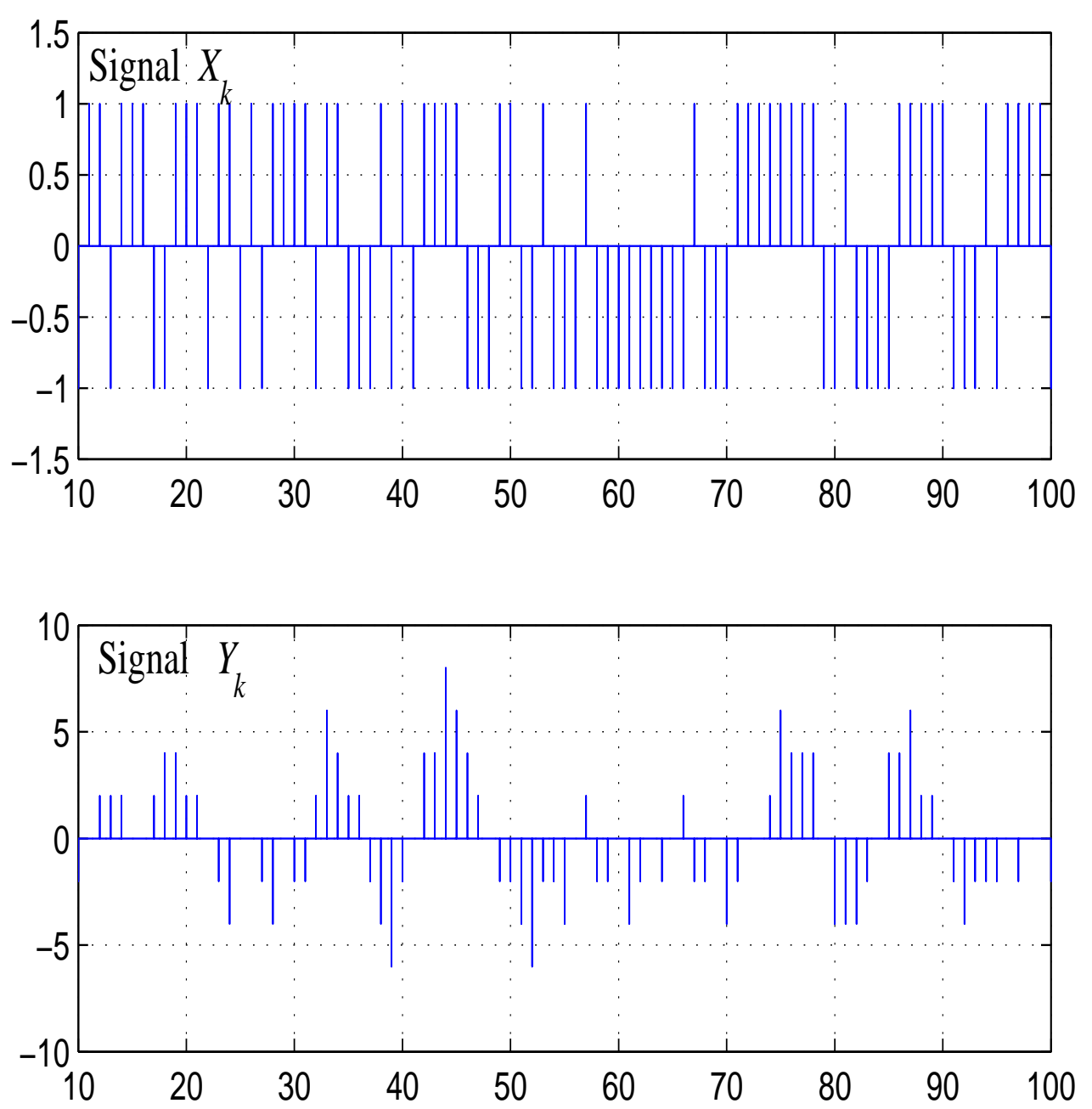

Fig. 9. Trajectories of two signals with Bernoulli input $W_{k} . X_{k}: \operatorname{MARC}(10)$ signal; $Y_{k}: \operatorname{MA}(10)$ with the same coefficients. 